ZOOLOGIE. - Observalions sur les Epyornis de Madagascar; par Ma. A. Minne-Enwards et Alfred Grandideb.

" Les découvertes qui ont èté faites récemment à Madagascar fournissent des renseignements nouveaux sur l'histoire des Epyornis. Les premières notions que nous ayons eues relativement à ces oiseaux gigantesques datent de I 85 I; elles sont dues à I. Geoffroy Saint-Hilaire qui fit alor's connaître des œufs et quelques fragments osseux trouvés dans le sud, près du port des Masikoro (Machicora). L'ètude de ces pièces permit à ce savant zoologiste de classer l'Epyornis parmi les Brévipennes, mais elle ne porta cependant pas la conviction dans tous les esprits, car Valencienes, loin d'admettre le rapprochement proposé par Geoffroy, regardait l'Epyornis comme une sorte de Pingouin et Bianconi voulait voir en lui un grand Rapace et peut-être l'oiseau Roc dont parle Marco Polo.

" L'un de nous, pendant une exploration de Madagascar, fit faire des fouilles dans un terrain marécageux de la côte ouest, à Ambolisatra ou Amboulitsatre, et il y découvrit plusieurs os du même oiseau qui furent l'objet d'un travail publié en 1869 et dans lequel nous montrâmes que l'Epyornis constitue, parmi les Brévipennnes, un type caractérisé par ses formes massives et par ses pattes énormes. Nous avons aussi reconnu que les ossements d'Ambolisatra provenaient de trois espèces distinctes : l'Epyornis maximus, I' $E$. medius et l' E. modestus.

"De nouveaux matériaux d'étude viennent de nous arriver. Ils ont été recueillis sur divers points de Madagascar. La collection la plus importante a été faite au centre même de l'île, à Antsirabé (' ), par M. Georges Muller

( ${ }^{1}$ ) Antsirabé est une ville située à 3 journées de marche de Tananarive à $125^{\mathrm{km}}$ environ dans le sud-sud-ouest par $19^{\circ} 52^{\prime} 45^{\prime \prime}$ de latitude sud et $44^{\circ} 40^{\prime} 00^{\prime \prime}$ de longitude est, à $1500^{\mathrm{m}}$ au-dessus du niveau de la mer; les petites plaines au milieu desquelles elle se trouve entre le Sahalambo et le Sahatsio, affluents du Manandena qui va se jeter dans la Mania, comme toutes celles qui existent dans cette partie du grand massif central, étaient jadis des lacs que le temps a desséchés. A côté d'Antsirabé, il y a plusieurs sources thermales, dont les eaux, très calcaires, ont une température de $+37^{\circ}$ et $+46^{\circ}$ et déposent des concrétions abondantes qu'exploitent de nombreux forçats malgaches pour en faire de la chaux avec laquelle on construit les principaux édifices de la capi-

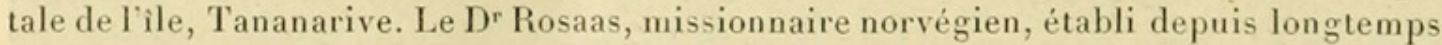
dans cette région, s'est employé avec la plus grande obligeance à aider les recherches de M. G. Muller. 


\section{$\left({ }_{12} 3\right)$}

et envoyée au Muséum d'Histoire naturelle, avant son départ pour le nord. C'est pendant cette dernière expédition qu'il fut assassiné sur la route de Mojanga par une troupe de pillards Sakalaves ou Fahavalo. Il laissait son œuvre inachevée, mais il avait déjà rendu à la Science des services importants, et, si la mort ne l'avait pas arrèté, son nom aurait certainement pris place à còté des explorateurs les plus méritants.

" D'autres ossements nous sont parvenus par les soins de M. Samat, négociant à Mouroundava; ils avaient été recueillis dans le sud-ouest.

"Enfin, M. Grevé a découvert sur la còte ouest, entre Bélo et Mouroundava, de nouveaux gisements fossilifères, et il a procuré au Muséum de belles pièces de squelettes d' Epyornis. Ce sont ces matériaux qui nous permettent de faire une étude plus complète des oiseaux disparus de Madagascar et de reconnaitre qu'ils se rapportent à des espèces nombreuses et variées.

"Les Epyornis constituent une famille représentée par des formes très diverses; on en compte aujourd'hui au moins douze, les unes de grande taille, les autres de dimensions médiocres. Les premières avaient plus de $3^{\mathrm{m}}$ de hauteur, les autres ne dépassaient guère les dimensions de l'Outarde. Leurs caractères anatomiques permettent de les répartir en deux sections : celle des Epyornis, à pattes larges et massives, et celle des Mullerornis ('), a pattes plus fines et qui ressemblaient davantage, par leurs proportions, aux Casoars de la Nouvelle-Guinée ou aux Apteryx de la Nouvelle-Zélande.

"On aurait pu supposer que des différences légères dans les dimensions ou dans les proportions des pièces du squelette pouvaient être des caractères sexuels; les plus grands de ces os appartenant à des màles et les plus petits à des femelles. Cette hypothèse n’est pas vérifiée par les faits, ainsi qu'on peut s'en convaincre en examinant la série des os de la jambe envoyés par M. Muller. Il en existe plas de soixante, ramassés dans la même localité, dont trente environ proviennent doiseaux adultes. Ces derniers sont, à peu de chose près, de taille égale et de proportions semblables; on ne peut admettre que, dans une série aussi considérable où sont représentés tous les àges, il ne se soit rencontré que des individus de mème sexe. Nous pouvons en conclure que les mâles et les femelles étaient semblables en taille, el, s'il en est ainsi pour une espèce d'Epyornis, il

( $\left.{ }^{1}\right)$ Nous proposons cette dénomination afin de conserver le souvenir de M. Muller, qui, le premier, nous a fait connaitre ces oiseaus. 
doit en être de même pour les autres. Les différences de dimensions indiquent donc des différences spécifiques.

" Genre Epyonnis. - Le plus grand de tous les Epyornis que nous appellerons $E$. ingens fait partie de la collection Grevé. Il surpassait de beaucoup l'E. maximus. Le fémur massif et court se fait surtout remarquer par la grosseur de la diaphyse, rappelant, d'une manière exagérée, les caractères que nous avons signalés chez l'espèce que nous venons de nommer et différant du même os chez l' $E$. Titan décrit par M. Andrews. Le tibia est d'une puissance extraordinaire et l'os du pied est particulièrement élargi dans ses parties articulaires (').

"De nombreux ossements de l'E. Titan (Andrews) nous ont aussi été envoyés de la côte ouest par M. Grevé et du sud par M. Samat; ils indiquent une espèce aussi haute mais moins massive et à articulations plus fines. L'E. maximus (I. Geoffroy) est notablement plus petit que les deux précédents et il se rapproche plus par ses proportions de l' $E$. ingens que de l' $E$. Titan; I'E. medius et le modestus doivent, dans une classification zoologique, prendre place à côté de l'espèce décrite par I. Geoffroy, mais leur taille est moindre $\left({ }^{2}\right)$.

"Deux autres espèces de fortes dimensions sont faciles à distinguer par les caractères de l'os du pied; l'une d'elles, Epyornis cursor $\left({ }^{3}\right)$, est presque égale à l' E. maximus, mais elle est plus grèle. L'autre, E. lentus ('), est remarquable par ses pieds courts et massifs.

"La nouvelle espèce que nous devons aux recherches de M. G. Muller et que nous nommerons $E$. Mulleri est plus petite; elle présente cependant des dimensions supérieures à celles de l' $E$. Hildebrandii décrit par M. Burckhardt et provenant aussi d'Antsirabé. Nous possédons le squelette

(1) Le corps du fémur dans sa portion la plus étroite mesure $29^{\mathrm{cm}}$ de circonférence sa largeur est de ${ }^{1} 0^{\mathrm{cm}}$. Le tibia a $8_{\mathrm{I}^{\mathrm{cm}}}$ de longueur, la circonférence de la diaphyse est de $20^{\mathrm{cm}}, 5$ (au plus étroit). Le tarso-métatarsien présente les dimensions suivantes : longueur $42^{\mathrm{cm}}$, largeur de l'extrémité supérieure $18^{\mathrm{cm}}$, circonférence du corps de l'os (au plus étroit) $2 \mathrm{I}^{\mathrm{cm}}, 5$, largeur au mème point $9^{\mathrm{cm}}$.

$\left({ }^{2}\right)$ Nous avons déjà fait connaître les caractères de cette espèce et indiqué les dimensions des principaux ossements dans un Mémoire publié en 1869.

$\left({ }^{3}\right)$ Longueur du tarso-métatarsien $38^{\mathrm{cm}}$, largeur de l'extrémité supérieure $14^{\mathrm{cm}}$, largeur de l'extrémité inférieure $12^{\mathrm{cm}}$, circonférence du corps de l'os $15^{\mathrm{cm}}, 5$, largeur $6^{\mathrm{cm}}, 5$.

(*) Longueur du tarso-métatarsien $36^{\mathrm{cm}}$, largeur de l'extrémité supérieure $15^{\mathrm{cm}}$, circonférence du corps de l'os $17^{\mathrm{cm}}$, largeur $6^{\mathrm{cm}}$ à $8^{\mathrm{cm}}$. 
presque entier de cet oiseau, le cràne, le bec inférieur, les vertèbres, les côtes, le sternum et ses annexes, une partie du bassin, les os des pattes et quelques phalanges, ce qui permet d'apprécier très exactement les affinités des Epyornis.

"La tête était moins aplatie que celle des Dinornis et beaucoup plus longue et plus étroite. Le cerveau était plus volumineux. Le condyle articulaire occipital est fortement pédonculé. Les fosses temporales sont profondes, mais étroites. Le basisphénoïde porte de chaque côté une apophyse ptérygoïdienne bien marquée. Le bec inférieur est droit, robuste, rappelant un peu par sa forme celui des Nandous ou Rhea, mais les branches maxillaires sont plus hautes, plus robustes. La portion symphysaire est longue, resserrée et excavée en forme de cuiller. Le sternum offre beaucoup de rapports avec celui des Apteryx ; c'est un plastron mince, aplati et très élargi; les surfaces articulaires coracoïdiennes sont disposées comme dans le genre néo-zélandais. Les coraco-scapulaires sont faibles et portent une très légère empreinte articulaire indiquant la présence d'un os du bras rudimentaire. Les pattes, toutes proportions gardées, ressemblent à celles de l' $E$. Tilan (').

"Genre Mullerornis. - Ces oiseaux, de taille moyenne, n'avaient pas l'apparence lourde et massive des Epyornis, ils se rapprochaient davantage des Casoars. Nous ne les connaissons encore que par quelques-uns des os de leur patte. Mais ces pièces permettent déjà de reconnaître trois espèces différentes.

"Le Mullerornis Betsilei vivait dans le centre de l'ìle, à côté de l' E. Mulleri; il était beaucoup moins abondant. L'os de la jambe est grêle, l'os du pied n'est pas élargi comme celui du genre précédent, et la section de la diaphyse figure un triangle presque isoscèle $\left({ }^{2}\right)$.

"Le Mullerornis agilis habitait la côte sud-ouest; nous n'avons de lui

( $\left.{ }^{1}\right)$ Longueur du fémur $32^{\mathrm{cm}}$, circonférence $19^{\mathrm{cm}}$, largeur $6^{\mathrm{cm}}, 5$. Longueur du tibia $54^{\mathrm{cm}}$, circonférence $\mathrm{I} 3 \mathrm{~cm}, 5$, largeur $4^{\mathrm{cm}}, 9$, largeur de l'articulation supérieure $10^{\mathrm{cm}}, 5$. Largeur de l'extrémité inférieure : $9^{\mathrm{cm}}, 5$. (Chez le $E$. Hildebrandti le tibia ne mesure que $48^{\mathrm{cm}}, 5$ et la circonférence du fémur n'est que de $15 \mathrm{~cm}, 8$.) Circonférence du tarso-métatarsien $\mathbf{I}_{4}^{\mathrm{cm}}, 5$, largeur $5^{\mathrm{cm}}, 8$, largeur de l'extrémité supérieure $\mathrm{I}^{\mathrm{cm}}$.

$\left(^{2}\right)$ Mullerornis Betsilei, longueur du tibia à partir de la surface articulaire fémorale $39^{\mathrm{cm}}$, circonférence de l'os $9^{\mathrm{cm}}$, largeur $3^{\mathrm{cm}}$, largeur de l'extrémité articulaire supérieure $7^{\mathrm{cm}}, 5$, largeur de l'extrémité inférieure $6^{\mathrm{cm}}$, longueur du tarso-métatarsien $3 \mathrm{I}^{\mathrm{cm}}(?)$, circonférence $8^{\mathrm{cm}}$, largeur du corps de $\mathrm{l}^{\prime} \mathrm{os} 2^{\mathrm{cm}}, 7$, largeur de l'extrémité supérieure $7^{\mathrm{em}}$. 


$$
\text { ( } 126)
$$

qu'un tibia remarquable par la manière dont les crètes osseuses intermusculaires et les coulisses tendineuses sont marquées. Le bord externe de l'os, au-dessus de l'articulation inférieure, se développe en une crête particulièrement saillante (').

" La troisième espéce ou M. rudis, a été trouvée par M. Grevé, dans les gisements de la côte ouest. Le tibia est à peu près de même longueur que celui du Mullerornis Betsilei, mais il est plus massif. Le tarso-métatarsien est remarquable par l'élargissement de l'extrémité inférieure, dont les poulies digitales sont très grosses. Entre la médiane et l'externe se trouve un pertuis osseux pour le passage du tendon du muscle adducteur du doigt externe; perluis qui n'existe pas chez les Epyornis $\left({ }^{2}\right)$.

1 Les conditions dans lesquelles ont été enfouis les restes de ces oiseaux semblent indiquer que les Epyornis el les Mullerornis fréquentaient les bords des eaux et que, s'ils ne nageaient pas, ils se tenaient au milieu des roseaux bordant les lacs ou les rivières. En effet, partout où ils ont été signalés, leurs ossements sont associès à ceux des petits Hippopotames, des Crocodiles et des Tortues, c'est-à-dire d'animaux tout à fait aquatiques. Les Epyornis devaient vivre d'ordinaire dans les plaines basses et inondées; ils y nichaient aussi, comme on peut en juger par le nombre des pièces du squelette de très jeunes oiseaux que l'on y trouve en abondance.

n Nous ajouterons que nous avons pu reconnaître, parmi les ossements d'Antsirabé, des pièces provenant d'un grand Rallide voisin de l'Aphanap$\operatorname{tery} x$ et d'un Anséride plus grand que ceux qui habitent Madagascar, indiquant encore des espèces aquatiques disparues, de la même époque que les Epyornis el vivant dans des conditions analogues.

"Ces grands oiseaux ont été certainement contemporains de l'Homme; on voit, sur quelques-uns de leurs os, des entailles profondes et très nettes qui ont èté faites par des instruments coupants, probablenent pour détacher la chair. Sur un fémur d'Hippopotame, de la mème date, on remarque uu trou entamant toute l'épaisseur de l'os et produit évidemment de main d'homme.

(1) Mullerornis agilis, longueur du tibia, à partir de la surface articulaire supérieure, $14^{\mathrm{cm}}$, circonférence de l'os $9^{\mathrm{cm}}, 7$, largeur $3^{\mathrm{cm}}, 4$, largeur de l'extrémité supérieure $6^{\mathrm{cm}}, 5$, largeur de l'extrémité inférieure $7^{\mathrm{cm}}$.

$\left({ }^{2}\right)$ Mullerornis rudis, longueur du tibia à partir de la surface articulaire supérieure $40^{\mathrm{cm}}$, circonférence de l'os $10^{\mathrm{cm}}$, largeur $3 \mathrm{~cm}, 5$, largeur de l'extrémité inférieure $7^{\mathrm{cm}}, 5$. 
„Ces découvertes en font pressentir d'autres plus importantes encore qui jetteront un peu de lumière sur les origines de Madagascar; dès aujourd'hui il est impossible de ne pas être frappé des analogies que présentait alors la faune de cette île avec celle de la Nouvelle-Zèlande où, à une époque peu ancienne, vivaient en grand nombre des oiseaux gigantesques, les Dinornis, représentés par plus de vingt espèces. Ce rapprochement semble indiquer certaines relations entre ces terres, aujourd'hui séparées par une immense étendue de mer, et il est en accord avec les observations relatives à l'ancienne faune des îles Mascareignes. ”

M. A. Des Chorzenux, en présenlant à l'Académie un nouveau fascicule de son "Manuel de Minéralogie ", s'exprime comme il suit :

" J'ai l'honneur d'offrir à l'Académie le deuxième fascicule du second Volume de mon "Manuel de Minéralogie ", qui contient les familles des titanides, des tantalides, des niobides, des tungstides, des molybdides, des vanadides, des chromides, des tellurides, des antimonides, des arsénides et des phosphorides.

„ Comme dans le Volume précédent, j’ai donné, d’une manière aussi complète que possible, le recueil des angles calculés, comparés aux angles mesurés par différents auteurs. La détermination des propriétés optiques biréfringentes a été faite sur tous les cristaux transparents et j’ai signalé les anomalies optiques dont les plus remarquables sont celles de la pérowskite.

"La publication de ce fascicule a été retardée par les nombreuses observations personnelles qu'il contient, et c'est gràce au concours de M. Lacroix que j'ai pu le terminer.

„J'espère qu'il nous sera possible de publier un troisième Volume, qui completera mon Manuel. »

\section{NOMINA TIONS.}

I'Académie procède, par la voie du scrutin, à l'élection d'un Membre de la Section de Géographie et Navigation, en remplacement de feu M. l'amiral Pâris. 


\section{$2 \mathrm{BHL}$ Biodiversity Heritage Library}

Milne-Edwards, Alphonse and Grandidier, Alfred. 1894. "Observations sur les Æppyornis de Madagascar." Comptes rendus hebdomadaires des séances de l'Académie des sciences 118, 122-127. https://doi.org/10.5962/bhl.part.17595.

View This Item Online: https://www.biodiversitylibrary.org/item/23772

DOI: https://doi.org/10.5962/bhl.part.17595

Permalink: https://www.biodiversitylibrary.org/partpdf/17595

\section{Holding Institution}

Harvard University, Museum of Comparative Zoology, Ernst Mayr Library

\section{Sponsored by}

Harvard University, Museum of Comparative Zoology, Ernst Mayr Library

\section{Copyright \& Reuse}

Copyright Status: NOT_IN_COPYRIGHT

This document was created from content at the Biodiversity Heritage Library, the world's largest open access digital library for biodiversity literature and archives. Visit BHL at https://www.biodiversitylibrary.org. 